\title{
TITLE:
}

\section{Contraction and reexpansion of polymer thin films}

AUTHOR(S):

Miyazaki, T; Nishida, K; Kanaya, T

CITATION:

Miyazaki, T ...[et al]. Contraction and reexpansion of polymer thin films. Physical Review E 2004, 69(2): 022801.

ISSUE DATE:

2004-02

URL:

http://hdl.handle.net/2433/39906

RIGHT:

Copyright 2004 American Physical Society 
PHYSICAL REVIEW E 69, 022801 (2004)

\title{
Contraction and reexpansion of polymer thin films
}

\author{
T. Miyazaki ${ }^{1,2}$ K. Nishida, ${ }^{1}$ and T. Kanaya ${ }^{1, *}$ \\ ${ }^{1}$ Institute for Chemical Research, Kyoto University, Uji, Kyoto-fu 611-0011, Japan \\ ${ }^{2}$ Nitto Denko Corporation, 1-1-2 Shimohozumi, Ibaraki, Osaka-fu 567-8680, Japan
}

(Received 16 June 2003; revised manuscript received 13 October 2003; published 27 February 2004)

\begin{abstract}
We report x-ray reflectivity measurements on polystyrene thin films supported on silicon wafer. In annealing experiments, we found fast and slow contraction processes in the thin films above the glass transition temperature. The former is the normal relaxation (annealing) process observed in bulk, and the latter is unexpected and enhanced in thin films below $\sim 20 \mathrm{~nm}$. In addition, we found unexpected extremely slow reexpansion processes in the glassy state. These unexpected very slow processes are discussed in terms of lateral contraction and expansion processes driven by entropic changes at the interfaces and the difference of the expansivities between polystyrene and silicon wafer.
\end{abstract}

DOI: 10.1103/PhysRevE.69.022801

PACS number(s): 36.20.-r, 64.70.Pf, 65.60.+a, 68.60.Dv

\section{INTRODUCTION}

Extensive studies have been carried out on polymer thin films and polymer surfaces [1,2] because their properties are very different from the bulk and related to many important phenomena such as adhesion, wetting, and surface friction. Glass transition of thin films is one of the most interesting subjects because many properties such as mechanical and thermal properties change drastically at the glass transition temperature $T_{g}$. Aiming to elucidate the special nature of glass transition of thin films and/or surfaces, studies have been performed using many techniques such as ellipsometry, $\mathrm{x}$-ray and neutron reflectometry, positron annihilation, dielectric relaxation, Brillouin light scattering, and atomic force microscopy [3]. One of the most interesting but unusual properties is contraction of film thickness with increasing temperature in the glassy state (apparent negative thermal expansivity), which was first observed by Orts et al. [4] for polystyrene thin films below about $25 \mathrm{~nm}$ by $\mathrm{x}$-ray reflectivity. It was predicted that this is caused by unrelaxed structure due to lack of annealing [5]. In a previous paper [6], we have investigated annealing effects on thickness of deuterated polystyrene thin films using neutron reflectivity to confirm the prediction, and found that the contraction with temperature in the glassy state originates from unrelaxed structure due to lack of annealing. In addition, the previous study [6] implied that there is an unexpected very slow film contraction process above the glass transition temperature. In order to elucidate these interesting but unusual observations, we investigated thickness changes of polystyrene thin films supported on silicon substrates with various thermal histories using $\mathrm{x}$-ray reflectivity, especially focusing on very thin films below $\sim 20 \mathrm{~nm}$.

\section{EXPERIMENTAL}

In this study, we used polystyrene (PS) with molecular weight $M_{w}=3.03 \times 10^{5}$ (Polymer Source, Inc.) and the molecular weight distribution was $M_{w} / M_{n}=1.09$, where $M_{w}$

\footnotetext{
*Corresponding author. Email: kanaya@scl.kyoto-u.ac.jp
}

and $M_{n}$ are the weight-averaged and number-averaged molecular weights, respectively.

PS thin films were prepared on cleaned silicon (Si) (111) wafers by spin coating the toluene solutions at $2000 \mathrm{rpm}$. Film thickness was controlled varying the concentration.

XR measurements were performed using a home-built $\mathrm{X}$-ray reflectometer which is based on a conventional powder diffractometer. Refer to Refs. $[7,8]$ for the reflectometer and the data analysis used in this study. The sample was placed in a chamber with beryllium windows under vacuum. The sample temperature was controlled within $\pm 0.1 \mathrm{~K}$ during the measurements.

As-deposited PS thin films were introduced in the chamber and kept at $298 \mathrm{~K}$ for $1 \mathrm{~h}$ under vacuum to remove any residual solvents. XR measurements for PS thin films were performed in a temperature range from 298 to $423 \mathrm{~K}$ for every $5 \mathrm{~K}$, and hence one temperature scan from 298 to 423 $\mathrm{K}$ took about $7 \mathrm{~h}$. PS samples were not exposed to air after they were introduced in the chamber of the reflectometer.

\section{RESULTS AND DISCUSSION}

XR measurements were carried out on as-deposited films with various values of initial thickness $d_{0}$ from 298 to $423 \mathrm{~K}$, and then on the films after annealing at $423 \mathrm{~K}$ for various periods of annealing time. An example of the observed reflectivity as well as the fit is shown in Fig. 1 for a film with initial thickness $d_{0}=17.8 \mathrm{~nm}$ at various temperatures. The temperature dependence of thickness evaluated from the reflectivity is shown for films with $d_{0}=9.32$ and $53.61 \mathrm{~nm}$ in Figs. 2(a) and 2(b), respectively. The as-deposited film with $d_{0}=53.61 \mathrm{~nm}$ increases in thickness with temperature up to around $370 \mathrm{~K}$ when it begins to decrease. After showing a minimum at around $380 \mathrm{~K}$, it again increases with temperature. This behavior is similar to that observed when unrelaxed bulk sample is annealed: structural relaxation occurs when annealed at or slightly below $T_{g}$ [9]. The temperature $370 \mathrm{~K}$ at which the thickness begins to decrease is close to the bulk glass transition temperature $T_{g}(=373 \mathrm{~K})$, suggesting that the contraction between 370 and $380 \mathrm{~K}$ is caused by structural relaxation. The film annealed at $423 \mathrm{~K}$ for $2 \mathrm{~h}$ does not show the contraction at around $370 \mathrm{~K}$, confirming that it 


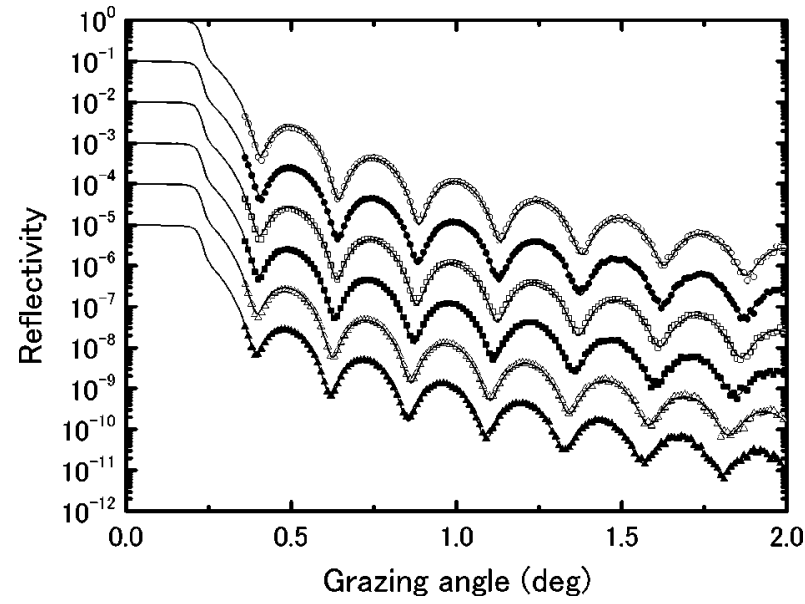

FIG. 1. X-ray reflectivities and the fitting results for a film with initial thickness $d_{0}=17.8 \mathrm{~nm}$ at $303(\bigcirc), 333(\bullet), 363(\square), 383$ $(\boldsymbol{\square}), 403(\triangle)$, and $423 \mathrm{~K}(\mathbf{\Delta})$ after annealing at $423 \mathrm{~K}$ for $2 \mathrm{~h}$.

is caused by unrelaxed structure due to lack of annealing. The thermal expasivities in the glassy and molten states are $1.1 \times 10^{-4}$ and $5.2 \times 10^{-4} \mathrm{~K}^{-1}$, respectively, which are almost the same as those expected from the bulk assuming that thin films are restrained along the substrate [10]. The glass transition temperature $T_{g}$ estimated from the change of thermal expansivity is $373 \mathrm{~K}$, which is also the same as the bulk value. These observations suggest that the glass transition

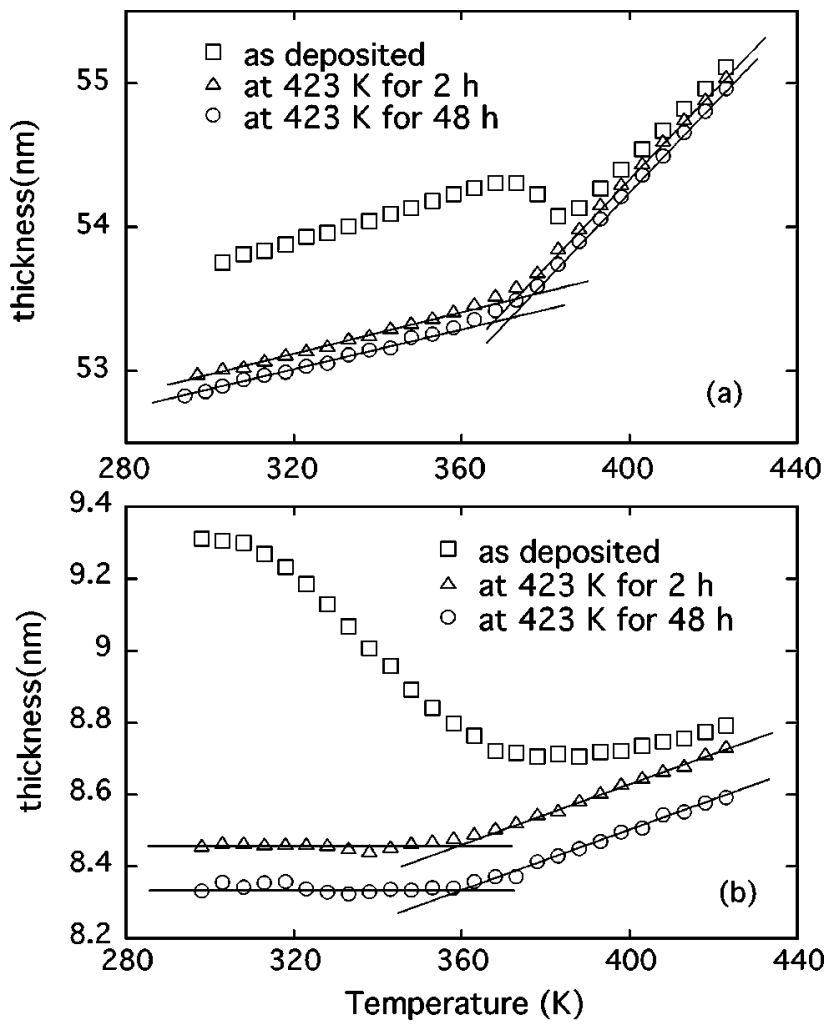

FIG. 2. Temperature dependence of thickness of films after annealing at $423 \mathrm{~K}$ for 2 and $48 \mathrm{~h}$. For comparison that of asdeposited films is also included. (a) Initial thickness $d_{0}$ $=53.61 \mathrm{~nm}$, (b) $d_{0}=9.32 \mathrm{~nm}$. behavior of the film with $d_{0}=53.61 \mathrm{~nm}$ is similar to the bulk. It is interesting to point out that further annealing at $423 \mathrm{~K}$ for $48 \mathrm{~h}$ does not affect either the thermal expansivities in the glassy and molten states or the glass transition temperature as seen in Fig. 2(a), but the thickness itself decreases with annealing time very slowly.

The temperature dependence of thickness for the asdeposited film with $d_{0}=9.32 \mathrm{~nm}$ is different from that with $d_{0}=53.61 \mathrm{~nm}$ [Fig. 2(b)]. The thickness is almost independent of temperature below $310 \mathrm{~K}$ while it begins to decrease steeply above $310 \mathrm{~K}$, suggesting onset of structure relaxation. A similar behavior is observed for films thinner than $\sim 20 \mathrm{~nm}$, and the onset temperature of structure relaxation becomes lower as the film thickness decreases. These observations for the thinner films qualitatively agree with those reported by Orts et al. [4]. After annealing at $423 \mathrm{~K}$ for $2 \mathrm{~h}$, the contraction below $310 \mathrm{~K}$ is not observed as seen in Fig. 2(b), confirming again that the contaction with temperature is caused by unrelaxed structure due to lack of annealing. The expansivities after annealing are $\sim 0$ and $5.3 \times 10^{-4} \mathrm{~K}^{-1}$ in the glassy and molten states, respectively. The latter is normal, but the former is very small compared with the value expected from the bulk although it is after annealing at 423 $\mathrm{K}$ for $2 \mathrm{~h}$ or more. For all well-annealed films thinner than $\sim 20 \mathrm{~nm}$, such a decrease in thermal expansivity is observed in the glassy state, suggesting that zero or very small expansivity is inherent nature of ultrathin films, probably less than $\sim 20 \mathrm{~nm}$. This problem will be discussed in a separated paper [11]. After $\sim 2 \mathrm{~h}$ annealing the expansivity and the glass transition temperature $T_{g}$ becomes independent of annealing time, but the thickness itself decreases extremely slowly with annealing time as observed for the film with $d_{0}=53.61 \mathrm{~nm}$.

As seen above, the structural relaxation due to annealing is almost completed within $\sim 2 \mathrm{~h}$ at $423 \mathrm{~K}$, but there is another very slow process reducing the thickness. In order to see this slow process the reduction in thickness $d(t)-d_{0}$ is plotted as a function of annealing time at $423 \mathrm{~K}$ in Fig. 3 for $d_{0}=88.6,53.6,11.7$, and $8.4 \mathrm{~nm}$. The thickness was measured at $298 \mathrm{~K}$ just after the annealing at $423 \mathrm{~K}$. After the fast contraction in the very early stage of annealing within two hours, thickness decreases extremely slowly with annealing time. In order to evaluate the relaxation time (or the contraction time) $\tau_{f}$ and $\tau_{s}$ we fitted the data to double exponential function $\left(d_{\infty}-d_{0}\right)\left[1-A_{f} \exp \left(-t / \tau_{f}\right)\right.$ $\left.-A_{s} \exp \left(-t / \tau_{s}\right)\right]$, where $d_{\infty}$ is the thickness at infinite annealing time and $A_{f}$ and $A_{s}$ are the fractions of the fast and slow processes. The solid curves in the figure are the results of fits. Note that $\tau_{f}$ in the fit to the data for $d_{0}=8.7 \mathrm{~nm}$ was fixed to be $1.9 \mathrm{~h}$, which is the average of all other samples, because the data points are few. The data points are rather scattered, so that the exact evaluation of the relaxation times is not easy. However, it is safe to say that the relaxation times of the fast and slow processes are 1-2 and 30-50 h for films thinner than $\sim 20 \mathrm{~nm}$, but that of the slow process is too long to be evaluated for films thicker than $\sim 20 \mathrm{~nm}$. The fast process is almost independent of thickness, suggesting that it is similar to a relaxation process observed for bulk. What is the slow process? The slow process is enhanced in 


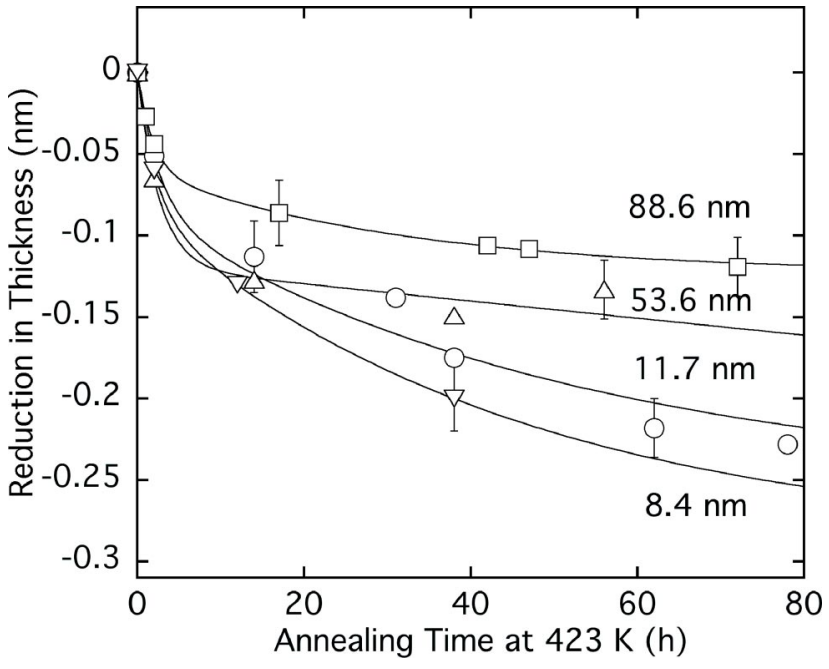

FIG. 3. Reduction in thickness as a function of annealing time at $423 \mathrm{~K}$ for various value of initial thickness $d_{0}=88.6(\square), 53.6(\triangle)$, $11.7(\bigcirc)$, and $8.4 \mathrm{~nm}(\nabla)$. Solid curves are the results of fits with $\left(d_{\infty}-d_{0}\right)\left[1-A_{f} \exp \left(-t / \tau_{f}\right)-A_{s} \exp \left(-t / \tau_{s}\right)\right]$.

films thinner than $\sim 20 \mathrm{~nm}$, indicating that the process is related to surface or confinement of chains. Furthermore, the relaxation time of $30-50 \mathrm{~h}$ at $423 \mathrm{~K}$ is too long for usual structural relaxation due to annealing [12]. One possible explanation of this slow process is the "sliding motion" in a thin film proposed by de Gennes [13] to explain the molecular weight dependence of glass transition temperature in thin freely standing films reported by Forrest et al. [14,15]. Regardless of the mechanism of the motion, what we have to consider is why the thickness decreases with time. Before considering the reason, we would like to introduce a surprising observation.

Open circles in Fig. 4 show the temperature dependence of thickness for the film with $d_{0}=6.33 \mathrm{~nm}$ after annealing at $423 \mathrm{~K}$ for $20 \mathrm{~h}$. The sample was further annealed at $423 \mathrm{~K}$ for more $20 \mathrm{~h}$ and cooled down to $298 \mathrm{~K}$ to measure the thickness. Because of the slow contraction process, the film thickness decreases to 6.23 or $0.06 \mathrm{~nm}$ lower than the initial value, which is shown by an open square in Fig. 4. This sample was kept at $298 \mathrm{~K}$ for about 1 day in vacuum and the thickness was again measured as a function of temperature from 298 to $423 \mathrm{~K}$. The result is shown in Fig. 4 by closed circles. It is surprising that the thickness increases to $6.29 \mathrm{~nm}$ at $298 \mathrm{~K}$. All the procedures were in vacuum and we have checked adsorption of oil mist from vacuum pump during the measurements. However, it is not the cause of the reexpansion. It is further surprising that the film does decrease in thickness upon heating similar to the as-deposited sample. After this temperature scan, the sample was again annealed at $423 \mathrm{~K}$ for further $8 \mathrm{~h}$ and cooled down to $298 \mathrm{~K}$. The thickness was $6.17 \mathrm{~nm}$ just after cooling down, which is shown by a closed square in Fig. 4. This value suggests that the annealing at $423 \mathrm{~K}$ for this $8 \mathrm{~h}$ canceled the reexpansion during the 1 day. In order to confirm the reexpansion again we continuously measured the thickness with time at $298 \mathrm{~K}$. The results are shown in inset. The thickness increases with time. This reexpansion process can be well fitted with the

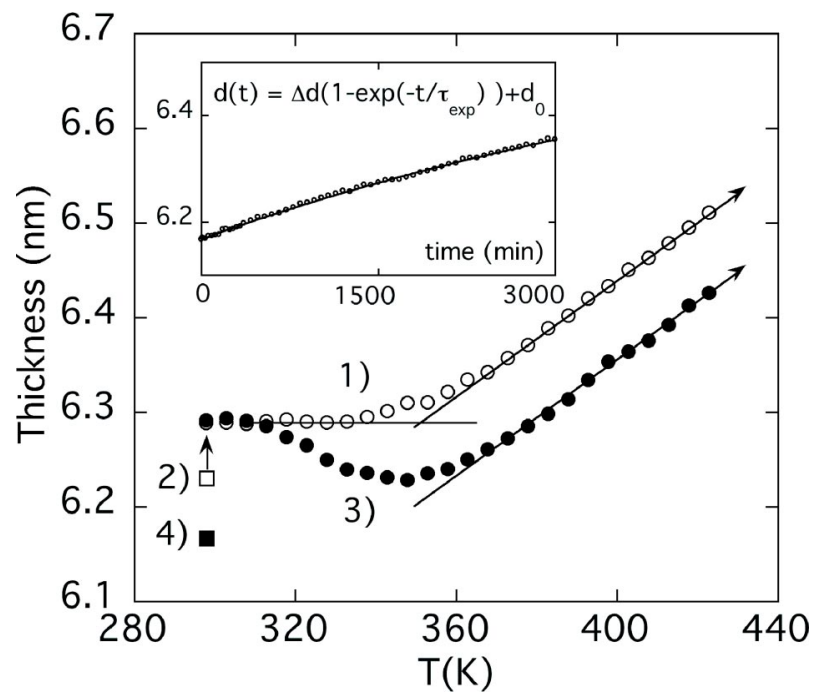

FIG. 4. Temperature dependence of thickness. (1) Temperature dependence of thickness after annealing at $423 \mathrm{~K}$ for $20 \mathrm{~h}(\bigcirc)$, (2) thickness at $298 \mathrm{~K}$ after further annealing at $423 \mathrm{~K}$ for more $20 \mathrm{~h}$ (40 h at $423 \mathrm{~K}$ in total) ( $\square$ ), (3) temperature dependence of thickness after keeping the sample at $298 \mathrm{~K}$ for 1 day ( ), (4) thickness at $298 \mathrm{~K}$ after further annealing at $423 \mathrm{~K}$ for more $8 \mathrm{~h}(48 \mathrm{~h}$ at 423 $\mathrm{K}$ in total) (ם). The inset shows time evolution of the film thickness at $298 \mathrm{~K}$ after cooling down from $423 \mathrm{~K}$ [after process (4)].

function $d(t)=\Delta d\left[1-\exp \left(-t / \tau_{\exp }\right)\right]+d_{0}$, where $\tau_{\text {exp }}$ is the expansion time. From the fit, it was found that the expansion time $\tau_{\text {exp }}$ is about 4 days and the film thickness almost recovers to the initial value $d_{0}$. Recently, Mukherjee et al. [16] also reported reexpansion of polymer thin films in the glassy.

In what follows, we would like to consider the physical origin of this surprising reexpansion at $298 \mathrm{~K}$ as well as the very slow contraction process at $423 \mathrm{~K}$ on the basis of an idea that these two processes could be lateral expansion and contraction of thin films. We consider thin polymer films with relaxed structure due to the fast annealing process and assume that there are fast and slow contraction processes as well as fast and slow expansion processes: both of the fast processes are usual thermal expansion and contraction processes due to vibration modes and responsible for the expansion and contraction normal to the film surface, and both of the slow processes are related to lateral expansion and contraction of the films.

As temperature increases the film increases in thickness along the direction normal to the surface due to usual (fast) expansion process. The lateral expansion is extremely suppressed due to restraint between the film and the substrate and the expansivity of $\mathrm{Si}$ is very small compared with polystyrene. It is based on the fact that thermal expansivity of a well-annealed film is the same as that expected from bulk assuming that polymer films are restrained on the surface of the substrate. Just after reaching a high temperature such as $423 \mathrm{~K}$ for polystyrene, the film expansion is completed only along the direction normal to the surface. This state must be unstable because polymer chains tend to form ordered structure exhibiting a layering [17] that is related to the radius of 
gyration of polymer [18] as pointed out by Mukherjee et al. [16], and hence the polymer chains may want to expand to the direction along the surface. However, this expansion is not easy because polymer chains are restrained on the substrate, and even if the chains take off the restraint of the substrate large scale motion along the surface is not easy for polymer chains. In thin films this motion must be the "sliding motion." Some works $[19,20]$ predicted that chain motion is diminished in thin films. However, it is not an origin of the slow contraction because it is enhanced in thinner films.

After the slow lateral expansion which may induce the vertical contraction, the thin film is cooled down to room temperature $(=298 \mathrm{~K})$ or $\sim 75 \mathrm{~K}$ below $T_{g}$, and contracts due to usual (fast) thermal contraction process along the direction normal to the surface. This state is also an unstable one because chains contract only normal to the surface, and hence they want to shrink to the surface direction. This process must be much harder than the slow lateral expansion in the melt because the chain mobility is much suppressed in the glassy state. However, in ultrathin films it is not impossible to move to the lateral direction owing to the "sliding motion" even at room temperature because ultrathin films have large fraction of surface. The contraction to the surface direction makes the film thicker because the density must be kept almost constant. From the fit to the reexpansion process (see inset in Fig. 4), we found that the initial thickness $d_{0}$ and the increment $\Delta d$ are 6.17 and $0.56 \mathrm{~nm}$, respectively. This means about $9 \%$ increase of volume if the lateral size of the film is constant. Such large increase of volume is impossible by reducing the density, implying that the contraction of the film along the lateral direction must occur. Thus, the unusual very slow re-expansion normal to the surface is observed in very thin films. The reexpanded films must be again in an unstable state because polymer chains are deformed due to anisotropic contraction during the slow lateral contraction process (or the vertical reexpansion process). This may be supported by the fact that the reexpanded films show the same contraction in the glassy state upon heating as the as-deposited films show (see Fig. 4).
[1] R. L. Jones and R. W. Richards, Polymers at Surface and Interfaces (Cambridge University Press, Cambridge, 1999).

[2] A. Karim and S. Kumar, in Polymer Surfaces, Interfaces and Thin Films (World Scientific, Singapore, 2000).

[3] See references in Refs. [1,2].

[4] W. J. Orts, J. H. v. Zanten, W. Wu, and S. K. Satija, Phys. Rev. Lett. 71, 867 (1993).

[5] J. A. Forrest and R. A. L. Jones, in Polymer Surfaces, Interfaces and Thin Films, (Ref. [2]), p. 251.

[6] T. Kanaya, T. Miyazaki, H. Watanabe, K. Nishida, H. Yamano, S. Tasaki, and D. B. Bucknall, Polymer 44, 3769 (2003).

[7] T. Miyazaki, A. Shiimazu, T. Matsushita, and K. Ikeda, J. Appl. Polym. Sci. 78, 1818 (2000).

[8] T. Miyazaki A. Shimazu, and K. Ikeda, Polymer 41, 8167 (2000).

[9] B. Frick, D. Richter, and C. Ritter, Europhys. Lett. 9, 557 (1989).

[10] W. E. Wallace, J. H. vanZanten, and W. Wu, Phys. Rev. E 52,

\section{R3329 (1995).}

[11] T. Miyazaki, K. Nishida, and T. Kanaya (unpublished).

[12] R. J. Roe and J. J. Curro, Macromolecules 16, 428 (1983).

[13] P. G. DeGennes, Eur. Phys. J. E 2, 201 (2000).

[14] J. A. Forrest, K. Dalnoki-Veress, and J. R. Dutcher, Phys. Rev. E 56, 5705 (1997).

[15] K. Dalnoki-Veress, J. A. Forrest, C. Murray, C. Gigault, and J. R. Dutcher, Phys. Rev. E 63, 031801 (2001).

[16] M. Mukherjee, M. Bhattacharya, M. K. Sanyal, T. Geue, J. Grenzer, and U. Pietsch, Phys. Rev. E 66, 061801 (2002).

[17] M. K. Sanyal, J. K. Basu, A. Datta, and S. Banerjee, Europhys. Lett. 36, 265 (1996).

[18] J. Kraus, P. Mueller-Buschbaum, T. Kuhlmann, D. W. Schubert, and M. Stamm, Europhys. Lett. 49, 210 (2000).

[19] X. Zheng, M. H. Rafailovich, J. Sokolov, Y. Strzhemechny, S. A. Schwarz, B. B. Sauer, and M. Rubinstein, Phys. Rev. Lett. 79, 241 (1997).

[20] A. N. Semenov, Phys. Rev. Lett. 80, 1908 (1998). 